Portland State University

PDXScholar

$11-9-2011$

\title{
Biomimetic broadband antireflection gratings on solar-grade multicrystalline silicon wafers
}

\author{
Blayne M. Phillips \\ University of Florida \\ Peng Jiang \\ University of Florida \\ Bin Jiang \\ Portland State University
}

Follow this and additional works at: https://pdxscholar.library.pdx.edu/mth_fac

Part of the Mathematics Commons

Let us know how access to this document benefits you.

\section{Citation Details}

Phillips, B. M., Jiang, P., \& Jiang, B. (2011). Biomimetic broadband antireflection gratings on solar-grade multicrystalline silicon wafers. [Article]. Applied Physics Letters, 99(19), 3.

This Article is brought to you for free and open access. It has been accepted for inclusion in Mathematics and Statistics Faculty Publications and Presentations by an authorized administrator of PDXScholar. Please contact us if we can make this document more accessible: pdxscholar@pdx.edu. 


\title{
Biomimetic broadband antireflection gratings on solar-grade multicrystalline silicon wafers
}

\author{
Blayne M. Phillips, ${ }^{1}$ Peng Jiang, ${ }^{1, a)}$ and Bin Jiang ${ }^{2}$ \\ ${ }^{1}$ Department of Chemical Engineering, University of Florida, Gainesville, Florida 32611, USA \\ ${ }^{2}$ Department of Mathematics and Statistics, Portland State University, Portland, Oregon 97201, USA
}

(Received 30 August 2011; accepted 24 October 2011; published online 9 November 2011)

\begin{abstract}
We report a simple and scalable bottom-up technique for fabricating broadband antireflection gratings on solar-grade multicrystalline silicon (mc-Si) wafers. A Langmuir-Blodgett process is developed to assemble close-packed silica microspheres on rough mc-Si substrates. Subwavelength moth-eye pillars can then be patterned on mc-Si by using the silica microspheres as structural template. Hemispherical reflectance measurements show that the resulting mc-Si gratings exhibit near zero reflection for a wide range of wavelengths. Both experimental results and theoretical prediction using a rigorous coupled-wave analysis model show that close-packed moth-eye arrays exhibit better antireflection performance than non-close-packed arrays due to a smoother refractive index gradient. (C) 2011 American Institute of Physics. [doi:10.1063/1.3660263]
\end{abstract}

Solar cells (or photovoltaics) produce electric power via conversion of the planet's most abundant and renewable energy input: sunlight. ${ }^{1}$ The production of photovoltaic panels is dominated by crystalline silicon solar cells. More specifically, 36\% of the 2004 production is based on single crystal silicon (sc-Si), $58 \%$ on multicrystalline silicon (mc-Si), and $4 \%$ on thin film amorphous silicon (a-Si). ${ }^{2}$ Although the conversion efficiency of mc-Si cells is lower than that of sc-Si cells, mc-Si panels are more popular due to their apparent cost benefits. Ideally, a solar cell should absorb all useful photons. However, due to the high refractive index of silicon, more than $30 \%$ of incident light is reflected back from the substrate. ${ }^{3}$ Vacuum-deposited quarterwavelength silicon nitride $\left(\mathrm{SiN}_{\mathrm{x}}\right)$ antireflection $(\mathrm{AR})$ coatings are widely used to suppress the unwanted optical reflection and improve the conversion efficiency of crystalline silicon photovoltaics. ${ }^{3}$ Unfortunately, traditional $\mathrm{SiN}_{\mathrm{x}}$ AR coatings suffer from high production cost, narrowband antireflection performance (i.e., they can only suppress reflection for a narrow range of wavelengths and incident angles), and poor thermal stability caused by the mismatch of thermal expansion coefficient between $\mathrm{SiN}_{\mathrm{x}}$ and $\mathrm{Si}$.

Inspired by the excellent antireflection properties of microstructured corneas of some nocturnal moths, ${ }^{4,5}$ broadband moth-eye AR gratings consisting of periodic arrays of subwavelength pillars have been extensively exploited by both top-down and bottom-up approaches. ${ }^{6-15}$ We have recently developed a scalable spin-coating technology that enables the wafer-scale production of non-closed-packed (NCP) colloidal monolayers which can then be used as template to create motheye gratings on flat sc-Si wafers. ${ }^{16,17}$ However, this promising technology cannot be extended to mc-Si substrates because the high surface roughness of solar-grade mc-Si wafers (see Figure 1(b)) impedes the formation of ordered colloidal template during spin-coating. Similarly, the high surface roughness hinders the fabrication of moth-eye AR gratings by most of the available top-down and bottom-up technologies which typically require a flat substrate surface (e.g., a uniform photoresist layer is essential for lithographic patterning). ${ }^{4,10,11}$ Although some

a)Electronic mail: pjiang@che.ufl.edu. available techniques such as spray deposition could create colloidal template on rough substrates, ${ }^{18-20}$ reproducible fabrication of monolayer colloidal crystals with good crystalline quality is still challenging.

Here, we report a simple and scalable colloidal templating technology that enables the fabrication of broadband moth-eye AR gratings on rough mc-Si wafers. Monodispersed silica microspheres with $250 \mathrm{~nm}$ diameter are synthesized by the standard Stöber method. ${ }^{21}$ The as-synthesized silica spheres are purified by repeated centrifugation/redispersion cycles in ethanol and are finally redispersed in ethylene glycol with particle volume fraction of 0.20 . Solar-grade mc-Si wafers (p-type, $125 \times 125 \mathrm{~mm}$, University Wafers) with root mean square roughness of $\sim 0.89 \mu \mathrm{m}$ (provided by the vendor) are RCA-cleaned (immersed in a 1:1:7 mixture of hydrogen peroxide: ammonia hydroxide: de-ionized water at $70{ }^{\circ} \mathrm{C}$ for an hour) prior to use.

Using a clamp attached to a syringe pump (KD Scientific 780-230), the mc-Si wafer is vertically immersed in a Kimax crystallizing dish $(170 \times 90 \mathrm{~mm})$ containing de-ionized water. The silica/ethylene glycol suspension is then added dropwise to the surface of the water. The suspension is spread to form a thin layer floating on the surface of the water. With the gradual dissolving of ethylene glycol in water, silica microspheres are accumulated at the water-air interface due to the high surface tension of water $\left(72.75 \mathrm{mN} / \mathrm{m}\right.$ at $\left.20^{\circ} \mathrm{C}\right)$. The capillary action between neighboring silica microspheres can then organize the floating particles into close-packed (CP) monolayer colloidal crystals which exhibit striking iridescence caused by light diffraction. ${ }^{22}$ Once the entire surface is covered with silica microspheres, it is left for $10 \mathrm{~min}$ for the silica spheres to form a homogeneous colloidal crystal. The mc-Si wafer is then slowly withdrawn at a rate of $\sim 0.5 \mathrm{~mm} / \mathrm{min}$ controlled by the syringe pump. As the wafer is withdrawn, the floating monolayer colloidal crystal is transferred onto the substrate. This simple colloidal self-assembly technology does not require sophisticated equipment (e.g., a LangmuirBlodgett trough) ${ }^{15}$ to organize silica microspheres with diameter ranging from $\sim 70 \mathrm{~nm}$ to $\sim 30 \mu$ m over wafer-sized areas. In addition, our preliminary results show that this technique is 

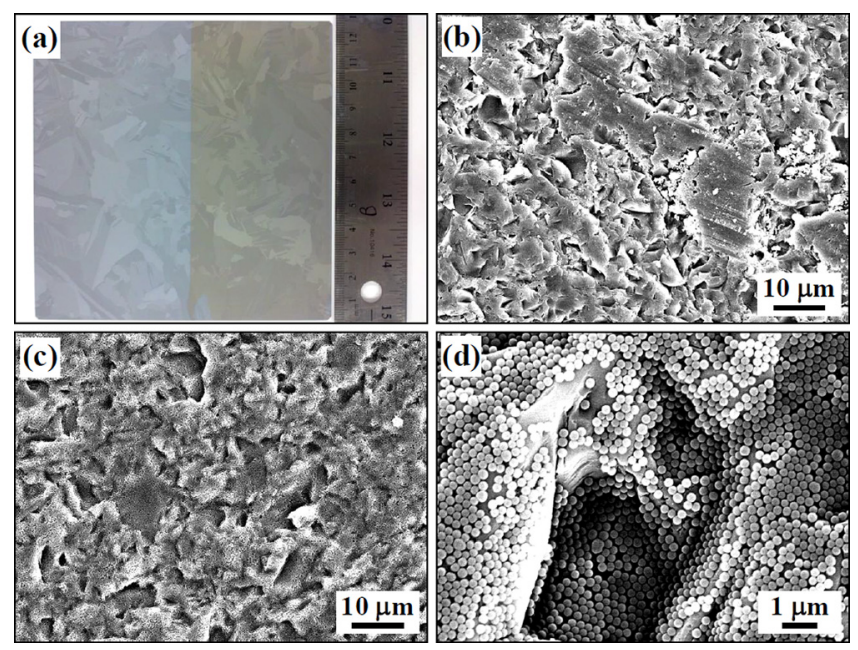

FIG. 1. (Color online) (a) Photograph of a commercial mc-Si wafer with the right half covered by a close-packed monolayer of $250 \mathrm{~nm}$ silica microspheres. (b) Typical top-view SEM image of a bare mc-Si wafer. (c) Typical top-view SEM image of the right part of the wafer in (a). (d) Magnified SEM image of (c).

compatible with roll-to-roll processing, promising for scaling up to large volume production.

Fig. 1(a) shows a photograph of a 5-in. solar-grade mcSi wafer with the right half (yellowish region) covered by a uniform monolayer of $250 \mathrm{~nm}$ silica microspheres. The typical top-view scanning electron microscope (SEM) images in Figs. 1(b) and 1(c) illustrate the left and right parts of the wafer. The high surface roughness of the wafer as evidenced by the randomly distributed, micrometer-sized pits and the uniform coverage of the rough surface by silica microspheres over large areas are clearly shown by these images. The magnified SEM image in Fig. 1(d) demonstrates that the hexagonal close-packing of the floating colloidal monolayers is retained during the colloidal transferring and drying processes. This is reasonable as the transferred colloidal monolayers are observed to float on a thin water wetting layer at the early stage of the particle transfer process. The high flexibility of this water layer renders the observed conformal coating of silica spheres on the rough mc-Si surface.
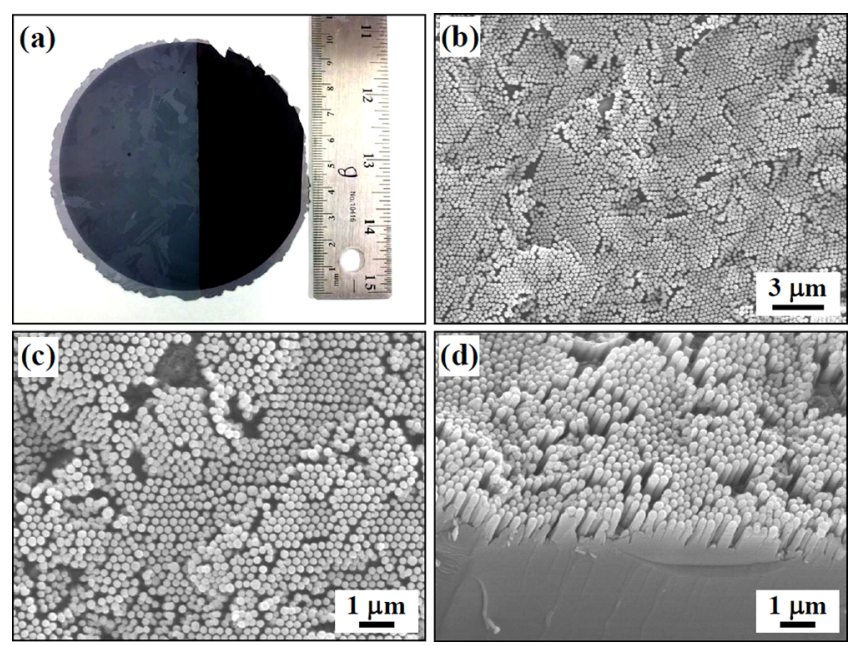

FIG. 2. (Color online) (a) Photograph of the mc-Si sample in Fig. 1(a) after chlorine RIE. (b) Typical top-view SEM image of the dark region in (a). (c) Magnified SEM image of (b). (d) Typical cross-sectional SEM image of the dark region in (a).
The close-packed silica microspheres can then be used as etching masks during a chlorine reactive ion etching (RIE) process (5 mTorr pressure, $20 \mathrm{SCCM}$ chlorine flow rate, and $80 \mathrm{~W}$ ) to create moth-eye AR gratings. As the etching rate of silica is much lower than that of silicon under the above RIE conditions, ${ }^{23}$ silica microspheres protect silicon immediately underneath them from being etched, resulting in the formation of pillar arrays directly on mc-Si wafers. Importantly, the different crystalline orientations (or domains) of mc-Si wafers do not affect the dry etching rate of silicon during chlorine RIE. ${ }^{23}$ The templating silica microspheres can finally be removed by dissolving in a 2 vol. \% hydrofluoric acid aqueous solution.

Fig. 2(a) shows a photograph of the wafer in Fig. 1(a) after 30 min RIE etching. The wafer here is cut to be circular to fit inside of our Unaxis Shuttlelock RIE/ICP (inductively coupled plasma) reactive-ion etcher chamber which requires samples to have a maximum diameter of 4-in. The dark part of the wafer is the area that is exposed to the reactive ions. The top-view SEM images in Figs. 2(b) and 2(c) show that hexagonally ordered pillars uniformly cover the dark region. The cross-sectional SEM image in Fig. 2(d) illustrates the close-packing of the templated pillars and the height of the pillars is determined to be $\sim 1 \mu \mathrm{m}$.

Spectral hemispherical reflectance measurements of the templated moth-eye AR gratings are carried out using a HR4000 UV-Vis spectrometer and an ISP-REF reflectance integrating sphere (both from Ocean Optics). Figure 3 compares the hemispherical reflectance obtained from a polished sc-Si wafer, a solar-grade mc-Si wafer, and the templated mc-Si grating in Fig. 2. The flat sc-Si wafer exhibits $30 \%-$ $50 \%$ hemispherical reflectance for wavelengths from 400 to $900 \mathrm{~nm}$, matching with early measurements in the literature. ${ }^{12,24}$ The rough surface of the commercial mc-Si facilitates to reduce the hemispherical reflectance to $20 \%-30 \%$. By contrast, the templated mc-Si grating shows excellent broadband antireflection property and the hemispherical reflectance is near zero for a wide range of wavelengths from $\sim 500 \mathrm{~nm}$ to $\sim 850 \mathrm{~nm}$.

The antireflection performance of the moth-eye AR gratings templated from CP colloidal crystals assembled at air-

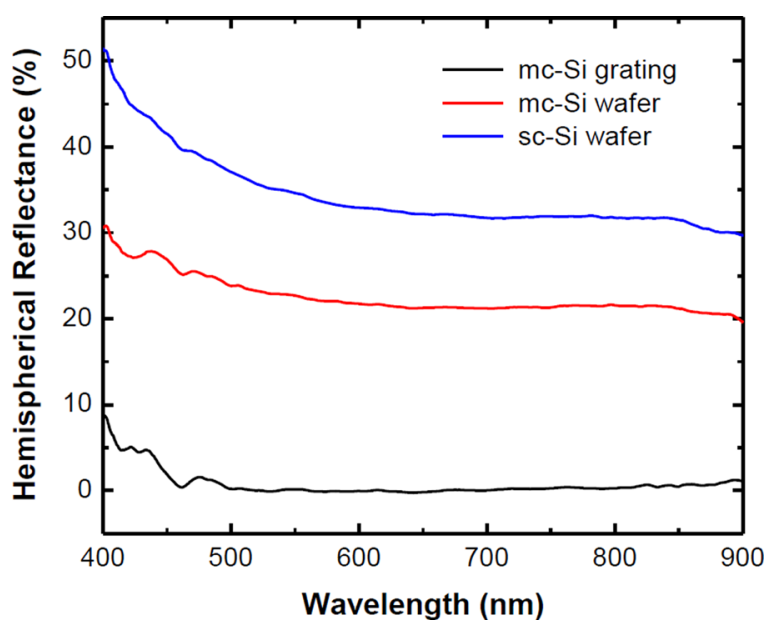

FIG. 3. (Color online) Comparison of hemispherical reflectance obtained from a polished sc-Si wafer, a commercial mc-Si wafer, and a templated mc-Si grating (dark region in Fig. 2(a)). 

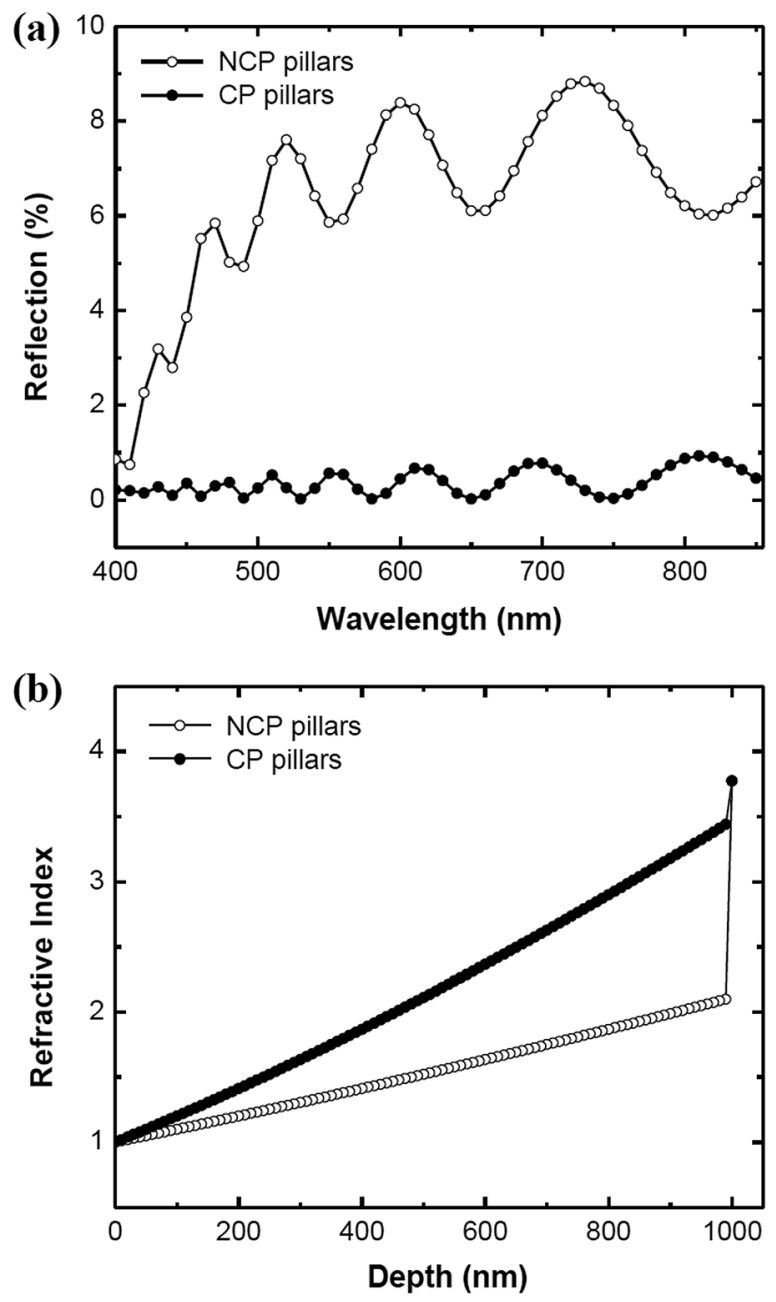

FIG. 4. (a) Comparison of the RCWA-simulated specular reflectance from a close-packed and a non-close-packed array of silicon pillars templated from $250 \mathrm{~nm}$ silica spheres. (b) Comparison of the calculated effective refractive index profile of a close-packed and a non-close-packed array of silicon pillars at $600 \mathrm{~nm}$ wavelength.

water interface is apparently better than that of spin-coatingderived AR gratings with NCP structure. ${ }^{16}$ A pillar height of $\sim 1 \mu \mathrm{m}$ is sufficient to create near zero reflection for subwavelength CP gratings, while a much larger optical depth $(>2 \mu \mathrm{m})$ is required to generate similar antireflection performance for NCP arrays. ${ }^{16}$ This significant height reduction can not only shorten the fabrication time of moth-eye AR gratings but can also reduce the surface area and the associated surface recombination of charge carries of the resulting solar cells. ${ }^{1}$ To gain insights into the effect of the $\mathrm{CP} / \mathrm{NCP}$ structure on the antireflection performance of moth-eye AR gratings, we have conducted theoretical calculations to simulate the specular reflectance of circular paraboloid nipple arrays using a rigorous coupled-wave analysis (RCWA) model. ${ }^{25}$

The calculation of effective refractive index and the reflectance of the whole system is described in our previous work. ${ }^{17}$ Here, the pillar lattice is assumed to be hexagonal and the distance between the centers of the neighboring troughs is defined as $\sqrt{2 s}$ for NCP (Ref. 16) or S for CP arrays, where $\mathrm{S}$ is the diameter of the templating silica spheres $(250 \mathrm{~nm}$ in this work). The base diameter of the pillars for both cases is assumed to be the same as the diameter of the silica spheres. Fig. 4(a) compares the simulated normal-incidence specular reflection of CP and NCP arrays with $1000 \mathrm{~nm}$ pillar height templated from $250 \mathrm{~nm}$ spheres. The reflection of the NCP array is apparently much higher than that of CP array. This is caused by the significant difference in the calculated refractive index profiles of the $\mathrm{CP}$ and NCP arrays as shown in Fig. 4(b). For the NCP array, the effective refractive index first changes gradually from 1.0 (air) to $\sim 2.1$ (at the bottom of the nipples) and then increases sharply to 3.774 (the index of silicon at $600 \mathrm{~nm}$ wavelength); ${ }^{26}$ while for the $\mathrm{CP}$ array, the refractive index changes much more smoothly from 1.0 to $\sim 3.44$ and then to 3.774. This smooth refractive index gradient leads to the very low reflection over a wide range of wavelengths. ${ }^{5}$

In summary, we have developed a simple yet scalable bottom-up technology for fabricating broadband AR gratings directly on rough mc-Si wafers. Optical measurements and RCWA simulations reveal that subwavelength gratings with CP structure exhibit improved antireflection performance than NCP arrays. Further structural optimization and integration of moth-eye AR gratings in mc-Si photovoltaic cells are underway and the optoelectronic properties of the final cells will be reported in our future publications.

This work was supported in part by DTRA and NSF under Grant Nos. CBET-0744879 and CMMI-1000686.

\footnotetext{
${ }^{1}$ A. Luque and S. Hegedus, Handbook of Photovoltaic Science and Engineering (Wiley, West Sussex, 2003).

${ }^{2}$ J. Poortmans and V. Arkhipov, Thin Film Solar Cells: Fabrication, Characterization and Applications (Wiley, Chichester, 2006).

${ }^{3}$ P. Doshi, G. E. Jellison, and A. Rohatgi, Appl. Opt. 36, 7826 (1997).

${ }^{4}$ P. B. Clapham and M. C. Hutley, Nature 244, 281 (1973).

${ }^{5}$ D. G. Stavenga, S. Foletti, G. Palasantzas, and K. Arikawa, Proc. R. Soc. London, Ser. B 273, 661 (2006).

${ }^{6}$ U. Schulz, Appl. Opt. 45, 1608 (2006).

${ }^{7}$ S. Chattopadhyay, L. C. Chen, and K. H. Chen, Crit. Rev. Solid State Mater. Sci. 31, 15 (2006).
}

${ }^{8}$ Q. Chen, G. Hubbard, P. A. Shields, C. Liu, D. W. E. Allsopp, W. N. Wang, and S. Abbott, Appl. Phys. Lett. 94, 263118 (2009).

${ }^{9}$ D. Lee, M. F. Rubner, and R. E. Cohen, Nano Lett. 6, 2305 (2006).

${ }^{10}$ Y. Kanamori, K. Hane, H. Sai, and H. Yugami, Appl. Phys. Lett. 78, 142 (2001).

${ }^{11}$ Y. Kanamori, M. Sasaki, and K. Hane, Opt. Lett. 24, 1422 (1999).

${ }^{12}$ Y. F. Huang, S. Chattopadhyay, Y. J. Jen, C. Y. Peng, T. A. Liu, Y. K. Hsu, C. L. Pan, H. C. Lo, C. H. Hsu, Y. H. Chang, C. S. Lee, K. H. Chen, and L. C. Chen, Nat. Nanotechnol. 2, 770 (2007).

${ }^{13}$ B. G. Prevo, E. W. Hon, and O. D. Velev, J. Mater. Chem. 17, 791 (2007).

${ }^{14}$ J. Zhu, Z. F. Yu, G. F. Burkhard, C. M. Hsu, S. T. Connor, Y. Q. Xu, Q. Wang, M. McGehee, S. H. Fan, and Y. Cui, Nano Lett. 9, 279 (2009).

${ }^{15}$ C. M. Hsu, S. T. Connor, M. X. Tang, and Y. Cui, Appl. Phys. Lett. 93, 133109 (2008).

${ }^{16}$ W. L. Min, B. Jiang, and P. Jiang, Adv. Mater. 20, 3914 (2008).

${ }^{17}$ C. H. Sun, P. Jiang, and B. Jiang, Appl. Phys. Lett. 92, 061112 (2008).

${ }^{18}$ A. Chunder, K. Etcheverry, S. Wadsworth, G. D. Boreman, and L. Zhai, J. Soc. Inf. Disp. 17, 389 (2009).

${ }^{19}$ W. Kern and E. Tracy, RCA Rev. 41, 133 (1980).

${ }^{20}$ G. M. Nogueira, D. Banerjee, R. E. Cohen, and M. F. Rubner, Langmuir 27, 7860 (2011).

${ }^{21}$ W. Stober, A. Fink, and E. Bohn, J. Colloid Interface Sci. 26, 62 (1968).

${ }^{22}$ N. D. Denkov, O. D. Velev, P. A. Kralchevsky, I. B. Ivanov, H. Yoshimura, and K. Nagayama, Nature 361, 26 (1993).

${ }^{23}$ M. J. Madou, Fundamentals of Microfabrication: The Science of Miniaturization, 2nd ed. (CRC, Boca Raton, FL, 2002).

${ }^{24}$ S. H. Zaidi, D. S. Ruby, and J. M. Gee, IEEE Trans. Electron Devices 48, 1200 (2001).

${ }^{25}$ M. G. Moharam, D. A. Pommet, E. B. Grann, and T. K. Gaylord, J. Opt. Soc. Am. A 12, 1077 (1995).

${ }^{26}$ M. A. Green and M. Keevers, Prog. Photovoltaics 3, 189 (1995). 